\title{
A High-Efficient Low-Cost Converter for Capacitive Wireless Power Transfer Systems
}

\author{
Il-Oun Lee ${ }^{1}$, Joongheon $\mathrm{Kim}^{2}$ and Woojoo Lee ${ }^{3, * \mathbb{D}}$ \\ 1 Department of Electrical Engineering, Myongji University, Yongin 17058, Korea; leeiloun@mju.ac.kr \\ 2 School of Computer Science and Engineering, Chung-Ang University, Seoul 06974, Korea; \\ joongheon@cau.ac.kr \\ 3 Department of Electronic Engineering, Myongji University, Yongin 17058, Korea \\ * Correspondence: spacelee@mju.ac.kr; Tel.: +82-10-2934-5835
}

Received: 19 August 2017; Accepted: 14 September 2017; Published: 18 September 2017

\begin{abstract}
Growth of the Internet of Things (IoT) spurs need for new ways of delivering power. Wireless power transfer (WPT) has come into the spotlight from both academia and industry as a promising way to power the IoT devices. As one of the well-known WPT techniques, the capacitive power transfer (CPT) has the merit of low electromagnetic radiation and amenability of combined power and data transfer over a capacitive interface. However, applying the CPT to the IoT devices is still challenging in reality. One of the major issues is due to the small capacitance of the capacitive interface, which results in low efficiency of the power transfer. To tackle this problem, we present a new step-up single-switch quasi-resonant (SSQR) converter for the CPT system. To enhance the CPT efficiency, the proposed converter is designed to operate at low frequency and drive small current into the capacitive interfaces. In addition, by eliminating resistor-capacitor-diode (RCD) snubber in the converter, we reduce the implementation cost of the CPT system. Based on intensive experimental work with a CPT system prototype that supports maximum $50 \mathrm{~W}(100 \mathrm{~V} / 0.5 \mathrm{~A})$ power transfer, we demonstrate the functional correctness of the converter that achieves up to $93 \%$ efficiency.
\end{abstract}

Keywords: capacitive wireless power transfer; single-switch quasi-resonant converter; wireless power transfer efficiency; low cost power transfer

\section{Introduction}

The rise of the Internet of Things (IoT) has been undergoing a paradigm shift on powering electronic devices. Traditional power transfers based on wired connection or battery replacement have hit a roadblock to support IoT devices that basically operate with tiny capacity batteries and are distributed everywhere. For example, sensor nodes in an IoT wireless sensor network cannot be deployed in hard-to-reach places where wiring is infeasible, batteries cannot be replaced or the need to replace the battery becomes very inconvenient. To overcome the drawback of the traditional power transfer systems, wireless power transfer (WPT) systems have been proposed. Expected to liberate the IoT devices from being wired or replacing batteries, the WPT systems have been receiving considerable interest from both academia and industry as the best-suited technique to power the IoT devices $[1,2]$.

According to the coupling method used in the WTP system, the WTP system can be classified into two types: inductive power transfer (IPT) and capacitive power transfer (CPT). The IPT is based on magnetic coupling between the winding coils, namely this technique uses the magnetic field to transfer power via air between resonators composed of the isolated windings [1,3,4]. On the other hand, the CPT is based on electric coupling between two metal plates, so that it uses electric field to transfer power through physical isolation barrier that utilizes the capacitance of capacitive interface [5-12]. 
Since the possible power transmission distance from the IPT is commonly longer than that of the CPT, the IPT seems to be predominantly used in WTP systems. However, the CPT systems have many important benefits that they are cheaper to implement, less vulnerable to metal barriers and electromagnetic interference (EMI), and more flexible with alignment than IPT systems [12]. Thanks to these advantages, the CPT systems have been adopted to various applications, such as biomedical microsystem [5], robot [13], and portable respiratory devices [14], rotating device [15,16] and so forth.

However, applying the CPT system to IoT devices is still challenging, mainly due to its low efficiency of the power transfer (i.e., scaling down the CPT systems to support small IoT devices operating with tiny capacity batteries is another challenging issue. This is, however, out of scope in this paper.) Because the primary concern of IoT devices has become energy efficiency [17], the low efficient CPT systems cannot be compromised with IoT devices. Therefore, it is strongly required to find solutions to enhance the efficiency of the CPT systems, which is the main objective of this paper. To do that, we perform an analysis in advance why the CPT systems lose efficiency. As a significant amount of power is dissipated from DC-DC converters in many other systems (e.g., multicore platforms [18,19], smartphones [20,21], and display systems [22]), the CPT converter in the CPT system is the main power consumer. Since the capacitance of the capacitive interface is limited by area availability of devices or systems, it is normally difficult to obtain the high capacitance of the capacitive interface [23]. The small capacitance of the capacitive interface requires an equipped CPT converter to operate at high resonant frequency, which results in significant increase of switching power loss. In addition, the small capacitance of the capacitive interface causes high equivalent series resistance (ESR) of the interface, which considerably increases conduction power loss. As a result, total power loss of the $\mathrm{CPT}$ converter results in critical efficiency degradation. More detailed elucidation regarding to this mechanism will be provided in Section 2 .

To minimize the power loss of the CPT converter and improve the efficiency of the CPT system, we propose a new step-up single-switch quasi-resonant (SSQR) converter. The proposed SSQR converter is designed to operate at low frequency so as to reduce the switching power loss, and to decrease the driving current into the capacitive interface in order to lessen the conduction power loss. In addition, we get rid of resistor-capacitor-diode (RCD) snubber in the converter, which results in retrenching implementation cost of the converter. Finally, a $50 \mathrm{~W}(100 \mathrm{~V} / 0.5 \mathrm{~A})$ prototype of the CPT system with the proposed SSQR converter is built to demonstrate the functional correctness and efficiency enhancement of the CPT system. To show the characteristics of the capacitive interface compared to film capacitors, the capacitive interface and a film capacitor are used as the resonant capacitor in the prototype system. The detailed analysis is performed with the prototype, which also takes consideration into the parasitic components of the system. From the intensive experimental work, the result shows that the proposed CPT system achieves the maximum efficiency of $93.93 \%$ at $20 \%$ load condition.

The remainder of this paper is organized as follows. Section 2 provides in-depth analysis of the CPT systems including its power loss mechanism. Section 3 presents the details of the proposed SSQR converter design. Sections 4 and 5 are dedicated for the experimental works that include operational analysis and experimental results based on the prototype development. Section 6 concludes the paper.

\section{Analysis of CPT Systems}

Since CPT converters in CPT systems exploit the capacitive interface to transfer power without wires and connectors, the capacitive interface is a core component of a CPT system. In general, the capacitance of the capacitive interface can be expressed as follows:

$$
C_{I 1}=C_{I 2}=\frac{A \cdot \epsilon_{0} \cdot \epsilon_{r}}{d}
$$

where $C_{I 1}$ and $C_{I 2}$ indicate the capacitances of two pairs of primary and secondary coupling plates (capacitive interfaces) $[8,24] ; A, \epsilon_{0}, \epsilon_{r}$, and $d$ denote the area of the capacitive interface, 
dielectric constant, relative dielectric constant, and distance between metal plates, respectively. $C_{I 1}$ and $C_{I 2}$ strongly affect the efficiency of a CPT converter, in that the higher $C_{I 1}$ and $C_{I 2}$ are preferred to design a high efficiency converter in a CPT system. From Equation (1), it is obvious that the capacitance of the capacitive interface is directly affected by $A$. Unfortunately, $A$ is limited by the available form factor of CPT systems. This is why a number of CPT systems report that $C_{I 1}$ and $C_{I 2}$ are in the order of a few hundred picofarad or a few nanofarad $[10,14,16,24]$. For example, the capacitance between metal plates with a $1 / 4 \mathrm{~mm}$ air gap in [23] is only $3.5 \mathrm{pF} / \mathrm{cm}^{2}$. Figure 1 shows the series LC tank in a CPT system that is composed of $L_{r}, C_{I 1}$ and $C_{I 2}$, where $L_{r}$ is a resonant inductor that is inserted in series into the capacitive interface to compensate its reactance.

Meanwhile, the capacitive interface suffers from a voltage stress ( $V_{C I 1}$ for the primary interface and $V_{C I 2}$ for the secondary interface) that may be expressed as:

$$
V_{C I 1}=\frac{I_{C I}}{2 \pi \cdot f_{r} \cdot C_{I 1}} \text { and } V_{C I 2}=\frac{I_{C I}}{2 \pi \cdot f_{r} \cdot C_{I 2}}
$$

where $I_{C I}$ and $f_{r}$ are the capacitive interface current and the resonant frequency, respectively. In general, the capacitive interface has a strict breakdown limit of the voltage stress. From Equation (2), designing $f_{r}$ and $C_{I 1}\left(C_{I 2}\right)$ to be high, but $I_{C I}$ to be small is required in order not to exceed the breakdown limit. However, setting higher $f_{r}$ results in the increase of switching power loss and the need to use higher $L_{r}$. Instead of rising $f_{r}$, it should be better to increase $C_{I 1}\left(C_{I 2}\right)$ and/or decrease $I_{C I}$, which derives $V_{C I 1}\left(V_{C I 12}\right)$ reduction.

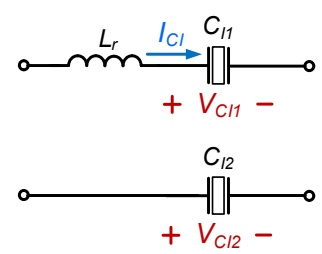

Figure 1. Capacitances of capacitive interface and series LC resonant tank in the CPT system.

From Equation (1), increasing $A$ may be the easiest way to increase $C_{I 1}\left(C_{I 2}\right)$. However, the bigger the size of CPT system is, the less versatile it will be. Instead, enhancing dielectric materials such as barium titanate $\left(\mathrm{BaTiO}_{3}\right)$, lead zirconium titanate (PZT), and polyethylene terephthalate (PET) can be used between the metal plates to increase $C_{I 1}\left(C_{I 2}\right)$. Unfortunately, such dielectric materials have high dielectric loss tangent $\tan \delta$, a.k.a. dissipation factor, which increases the equivalent series resistance (ESR) of the capacitive interface $R_{C I 1}$ and $R_{C I 2} . R_{C I 1}$ and $R_{C I 2}$ is described in Figure 2a and can be expressed as [25]:

$$
R_{C I 1}=R_{C I 2}=\frac{\tan \delta}{\omega \cdot C_{I 1}}=\frac{\tan \delta}{\omega \cdot C_{I 2}}
$$

where $\omega=2 \pi f_{r}$. The increased $R_{C I 1}$ and $R_{C I 2}$ generates more power loss (thereby more heat) in the capacitive interface, which is undesirable.

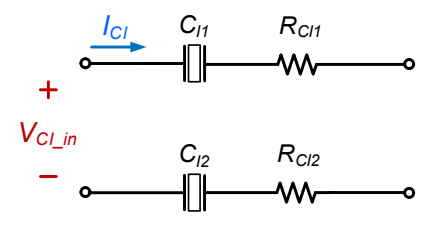

(a)

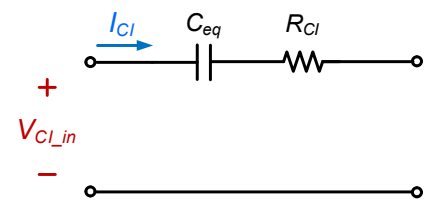

(b)

Figure 2. (a) capacitive interface and (b) its equivalent circuit in the CPT system. 
The difficulty to increase capacitance of the capacitive interface due to the technical and spacial limits induces inevitable power loss in the CPT system. The low capacitance of the interface increases $f_{r}$, thereby switching power loss increases. In addition, the low capacitance of the interface induces the high ESR, that, in turn, increases conduction power loss. More precisely, the switching power loss is linearly proportional to $f_{r}$ that can be formed:

$$
f_{r}=\frac{1}{2 \pi \sqrt{L_{r} \cdot C_{e q}}}
$$

where $C_{e q}\left(=C_{I 1} \| C_{I 2}\right)$ is the equivalent capacitance of the interface. The conduction loss is linearly proportional to the equivalent resistance $R_{e q}\left(=R_{C I 1}+R_{C I 2}\right)$ and square proportional to $I_{C I}$. From Equation (3), $R_{e q}$ increases with lowering $C_{I 1}$ and $C_{I 2}$. For reference, $C_{e q}$ and $R_{e q}$ are presented in Figure $2 \mathrm{~b}$. In addition, we notice that lowering $I_{C I}$ are helpful for not only reducing the voltage stress from Equation (2), but also save power from the conduction loss, i.e., by intuition, $I_{C I}$ can be decreased by increasing the input voltage of the capacitive interface $V_{C_{-} i n}$. Therefore, we focus on both $f_{r}$ and $I_{C I}$ as an optimization objective in the CPT system. The proposed CPT converter design in the following sections shows our achievement.

\section{Step-Up Single-Switch Quasi-Resonant Converter for CPT Systems}

The driving circuit topologies of the CPT system are generally classified into three types: (i) full-bridge (or half-bridge) inverter [8,9]; (ii) push-pull converter [13,24]; and (iii) single-switch topology with an AC coupling capacitor (Sepic, Zeta, Cuk, and buck-boost converters) [10]. Except for the single-switch topology with an coupling capacitor, full-bridge rectifiers are normally required as a rectifier circuit in the driving circuits. For low cost and high efficient CTP systems, the best-suit driving circuit type may be the single-switch topology with AC coupling capacitor, owing to its superiority in the less number of electric components and less power loss compared to the others. However, as aforementioned, this topology requires relatively large equivalent capacitance $C_{e q}$ for the low-frequency operation, which may result in still low efficiency of the power transfer.

To improve the efficiency of the single-switch topology with an AC coupling capacitor, we focus on a type of single-switch quasi-resonant (SSQR) converter. In general, the SSQR converter is composed of a switch $Q_{1}$, a transformer $T_{1}$, a resonant capacitor $C_{r}$, a resistive-capacitor-diode (RCD) snubber, two diodes $D_{1}$ and $D_{2}$, and an output filter $C_{O}$. Figure 3a shows the representative schematic of a step-down (buck) SSQR converter [26]. Thanks to the quasi-resonant operation, the SSQR converter reduces the turn-on and turn-off loss from $Q_{1}$ and alleviates the reverse-recovery losses from $D_{1}$ and $D_{2}$. The RCD snubber is used on the primary side to suppress the high voltage spike of $Q_{1}$ caused by the leakage inductance of $T_{1}$ and the high input voltage $V_{I N}$ at the switch turn-off transition. Especially in [26], two expensive multi-layer ceramic capacitor (MLCC) resonant capacitors with high capacitance of $22 \mu \mathrm{F}$ are used for $C_{r}$ in the secondary side, in order for low $f_{r}$.

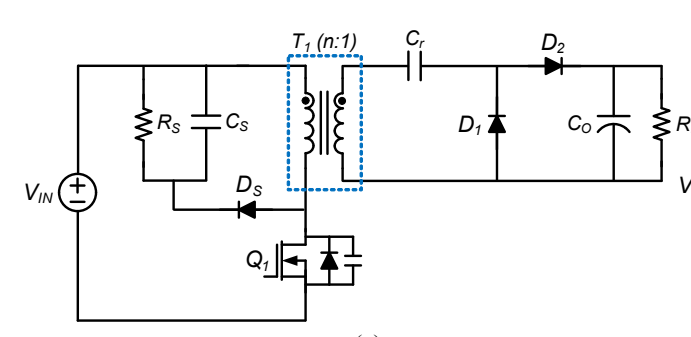

(a)

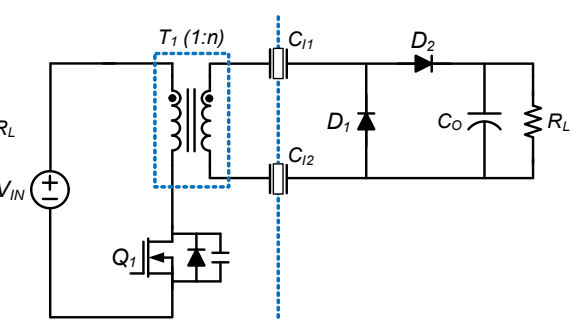

(b)

Figure 3. Schematics of the (a) step-down and (b) step-up single-switch quasi-resonant converter with a resonant capacitor. 
Since the voltage stress of $D_{1}$ and $D_{2}$ is clamped to the output voltage $V_{O}$, the SSQR converter is especially good at step-up (boost) applications. We thus design a step-up SSQR converter for the CPT system, wherein the capacitive interface is used for $C_{r}$. The schematic of the proposed converter is shown in Figure $3 \mathrm{~b}$. This converter operates with small $C_{e q}$ at low $f_{r}$, and drives small $I_{C I}$. Since the voltage overshoot at the switch turn-off transition is within a maximum drain-source voltage rating of $Q_{1}$, we eliminate the RCD snubber, thereby saving power. As a result, the step-up SSQR converter transfers power through the capacitive interface with high efficiency.

The proposed step-up SSQR converter is analyzed by taking account for the primary and secondary leakage inductances of $T_{1}, L_{l k 1}$ and $L_{l k 2}$, respectively, the output capacitor $C_{o s s}$ and the internal anti-parallel diodes of the switch $Q_{1}$, and the junction capacitor $C_{j}$ of $D_{1}$ and $D_{2}$ in detail. Figure 4 shows the schematics of the proposed SSQR converter to be analyzed. The validity of the step-up SSQR converter has been verified on a prototype that supports up to $50 \mathrm{~W}(100 \mathrm{~V} / 0.5 \mathrm{~A})$ power transfer. To show the characteristics of the capacitive interface, the capacitive interface for CPT systems and a film capacitor for general converters are investigated as a resonant capacitor in the proposed step-up SSQR converter. The results of the analysis and comparison of both capacitive interface and a film capacitor based step-up SSQR converters will be provided in Section 5 .

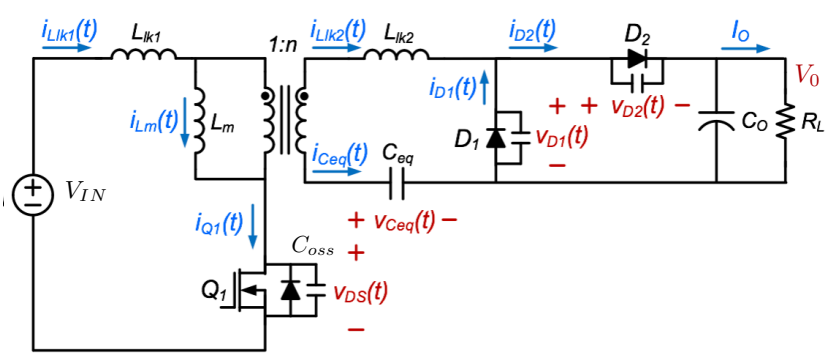

Figure 4. Current and voltage analysis of the proposed SSQR converter.

\subsection{Low Resonant Frequency}

By turning on the switch $Q_{1}$ in Figure 4, power is transferred from a voltage source to a load. Then, the resonant current flows through $T_{1}, Q_{1}, C_{e q}, D_{2}$ (not $D_{1}$ ) and the load. The components in the secondary side of $T_{1}$ can be transformed into their equivalences in the primary side by using the turn ratio $n$ of $T_{1}$. More precisely, the equivalence of the secondary leakage inductance $L_{l k 2}$ on the primary side is almost equal to the primary leakage inductance $L_{l k 1}$, thus the leakage inductance $L_{l k}$ equals $L_{l k 1}=L_{l k 2}$. The equivalent capacitance $C_{e q}$ of the capacitive interface is multiplied by $n^{2}$. The transformed schematics is shown in Figure 5a. As a result, the step-up turn ratio $n$ of the SSQR converter makes the small equivalent capacitance $C_{e q}$ of the capacitive interface look very large in the primary side, which results in the low resonant frequency $f_{r}$.

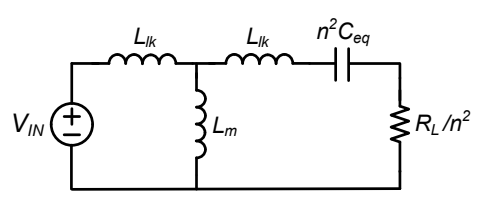

(a)

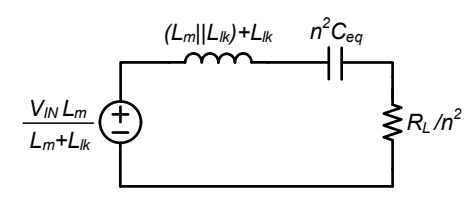

(b)

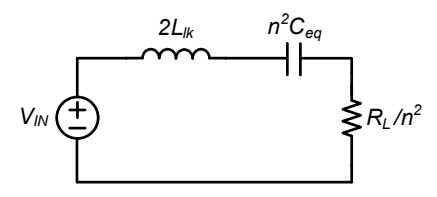

(c)

Figure 5. Steps to develop the equivalent circuit of the proposed SSQR converter in Figure 4, when the switch is turned on: (a) first, apply $L_{l k}=L_{l k 1}=L_{l k 2}, n^{2} C_{e q}$, and $R_{L} / n^{2} ;(\mathbf{b})$ next, merge $L_{m}$ and $L_{l k}$ to a single inductor; and finally (c) take account of $L_{m} \gg L_{l k}$.

Meanwhile, if the magnetizing inductance $L_{m}$ is much larger than $L_{l k}$, the equivalent circuit in Figure $5 a$ can be further simplified to the equivalent circuits as seen in Figure 5b,c. The input voltage and the resonant inductance of the resonant tank can be expressed as: 


$$
\left.\begin{array}{c}
\frac{L_{m} \cdot V_{I N}}{L_{m}+L_{l k}} \simeq V_{I N}, \\
\left(L_{m}|| L_{l k}\right)+L_{l k}=\frac{L_{m} \cdot L_{l k}}{L_{m}+L_{l k}}+L_{l k} \simeq 2 L_{l k},
\end{array}\right\} \quad\left(\text { if } L_{m} \gg L_{l k}\right) .
$$

From Equation (4), $f_{r}$ of the resonant tank of the proposed converter now can be reformulated to:

$$
f_{r}=\frac{1}{2 \pi \sqrt{2 L_{l k} \cdot n^{2} \cdot C_{e q}}}=\frac{1}{2 \pi \cdot n \sqrt{2 L_{l k} \cdot C_{e q}}} .
$$

Thanks to the low $f_{r}$, the step-up SSQR converter operates at a low switching frequency, thereby switching power loss should be reduced.

\subsection{Low Capacitive Interface Current}

Owing to the step-up turn ratio $n$ of the transformer $T_{1}$, the step-up SSQR converter powers the capacitive interface with low current and high voltage. The conduction power loss (a.k.a. $I^{2} R$ loss) of the capacitive interface is proportional to the square of the current but proportional to the ESR of the capacitive interface. Therefore, the effect of the the ESR increase due to the low $f_{r}$ (cf. from Equation (3)) to the conduction power loss can be canceled out from decreasing the capacitive interface current. Alternatively, the conduction loss can be further reduced by decreasing the capacitive interface current more aggressively, so that the step-up SSQR converter can transfer power through the capacitive interface with high efficiency.

\subsection{Elimination of the RCD Snubber}

The voltage overshoot occurs at the switch turn-off transition of the SSQR converter. When $Q_{1}$ is turned off, the resonant current flows through the transformer, the output capacitor $C_{\text {oss }}$ of the switch $Q_{1}, C_{e q}$, and diode $D_{1}$ (not $D_{2}$ ). Since this transition is very short, a function of the voltage across the equivalent capacitor for a certain time $t, V_{C_{e q}}(t)$ can be treated as the voltage at $t=0$, $V_{C_{e q}}(0)$. Like what we performed the simplification in Figure 5 by assuming $L_{m} \gg L_{l k}$, here we also can simplify the schematic of the SSQR converter when the switch is turned off to Figure 6. Due to the quasi-resonant operation, the primary leakage inductor current $i_{L l k 1}(t)$ and the secondary leakage inductor current $i_{L l k 2}(t)$ are low at the switch turn-off transition (cf. Figure 4). Therefore, the initial values of the leakage inductor currents are low. Plus, the step-up SSQR converter has the low $V_{I N}$ that is lower than $V_{O}$. Moreover, for the primary side of the transformer, $V_{C_{e q}}(0)$ and $L_{l k}$ may look small, due to the step-up turn ratio $n$. As a consequence, the voltage overshoot at the switch turn-off transition is within the maximum drain-source voltage rating of $Q_{1}$. We thus get rid of the RCD snubber, that, in turn, eliminates the corresponding power loss of the SSQR converter and reduces the number of components in the converter.

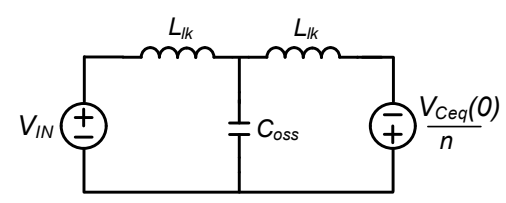

Figure 6. Equivalent circuit of the proposed SSQR converter when the switch is turned off.

\subsection{Low Component Counts}

The CPT converters are conventionally composed of a full-bridge inverter, a resonant inductor, capacitive interface, a full-bridge rectifier, and an output filter. Namely, at least four switches and four diodes are necessary to implement such converters. On the other hand, the proposed SSQR converter 
requires only a single switch and two diodes as semiconductor components. Therefore, the proposed converter has an edge in cost competitiveness compared to the conventional CPT converters.

\section{Operational Analysis}

The proposed SSQR converter has several operational modes in one switching cycle, which can be divided into eight modes according to time $t$. These eight operational modes are described in Figure 7. The characteristic waveforms corresponding to the eight operational modes are depicted in Figure 8. To facilitate the operational analysis with minimum loss of generality, we assume that (i) a switch $Q_{1}$ is ideal except for the inherent output capacitor $C_{\text {oss }}$ and the internal anti-parallel diodes; (ii) two rectifier diodes are ideal except for the junction capacitors $C_{j}$; and (iii) input voltage $V_{I N}$ and output voltage $V_{O}$ are constant. Note that, in Section 5 , the measured result of the operational modes with a prototype will be provided in detail.

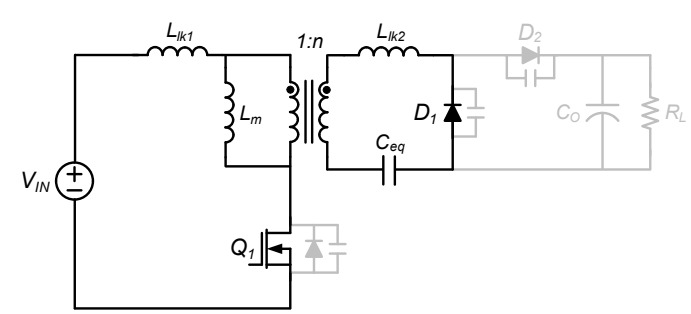

(a)

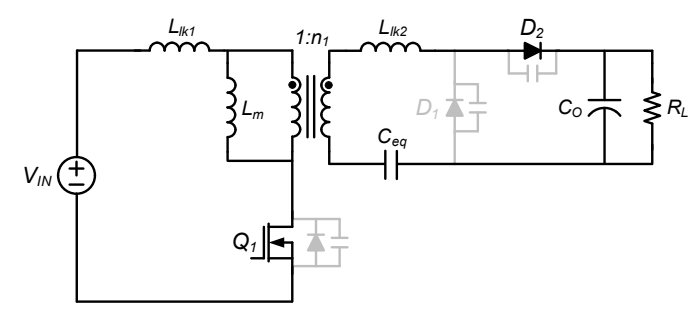

(c)

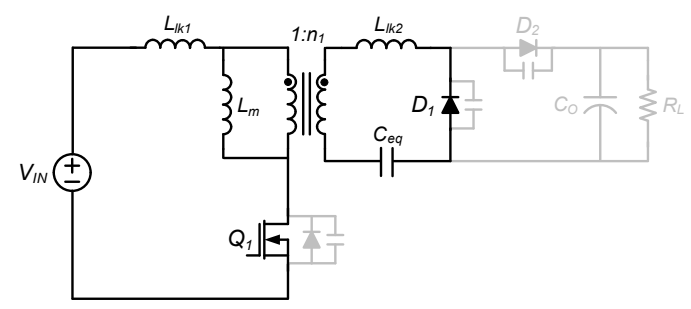

(e)

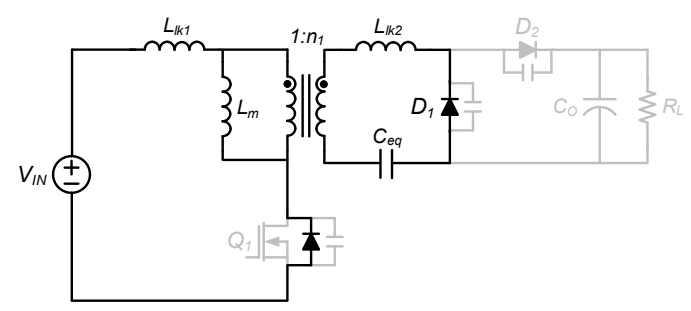

(g)

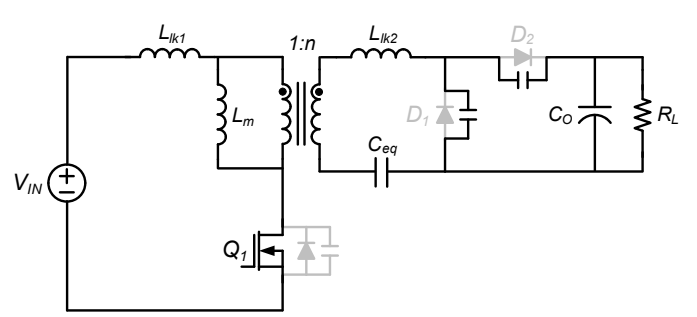

(b)

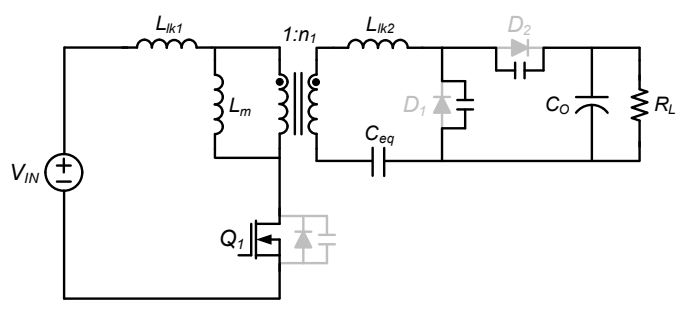

(d)

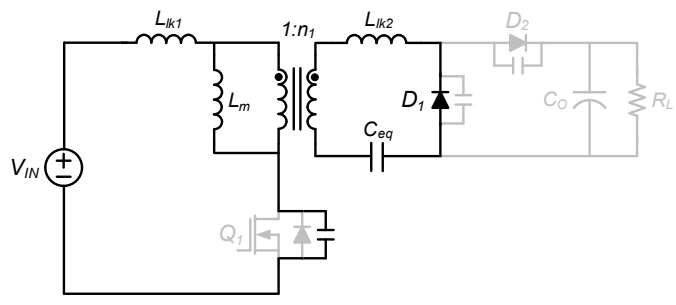

(f)

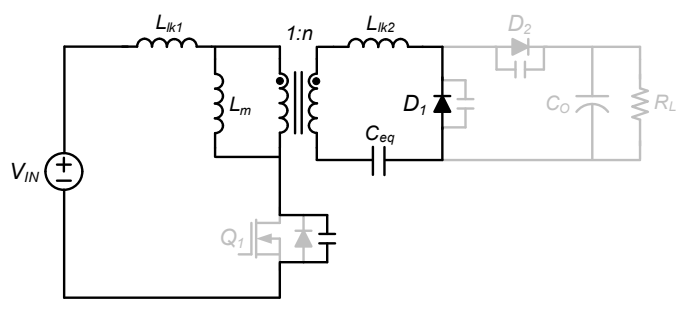

(h)

Figure 7. Operational analysis at $t$ : (a) Mode $1\left(t_{0}<t \leq t_{1}\right)$; (b) Mode $2\left(t_{1}<t \leq t_{2}\right)$; (c) Mode 3 $\left(t_{2}<t \leq t_{3}\right)$; (d) Mode $4\left(t_{3}<t \leq t_{4}\right)$; (e) Mode $5\left(t_{4}<t \leq t_{5}\right)$; (f) Mode $6\left(t_{5}<t \leq t_{6}\right)$; (g) Mode 7 $\left(t_{6}<t \leq t_{7}\right)$; and (h) Mode $8\left(t_{7}<t \leq t_{8}\right)$.

Before Mode $1\left(t \leq t_{0}\right)$ [Figure $7 \mathbf{h}$ ]: Before $t_{0}$, the switch $Q_{1}$ and the diode $D_{1}$ in Figure 4 are turned on. The current flows through the transformer, the output capacitor $C_{\text {oss }}$ of $Q_{1}$, the equivalent capacitance $C_{e q}$, and $D_{1}$. The inductances of the transformer resonates with the $C_{o s s}$. At the same time, 
the $V_{C_{e q}}(t)$ is applied to the transformer, and then the transformer is reset and $V_{C_{e q}}(t)$ starts to increase. As a result, the voltage across the switch $V_{D S}(t)$ increases with $V_{C_{e q}}(t)$ and resonates with inductances of the transformer. The primary leakage inductor current $i_{L l k 1}(t)$ is almost zero.

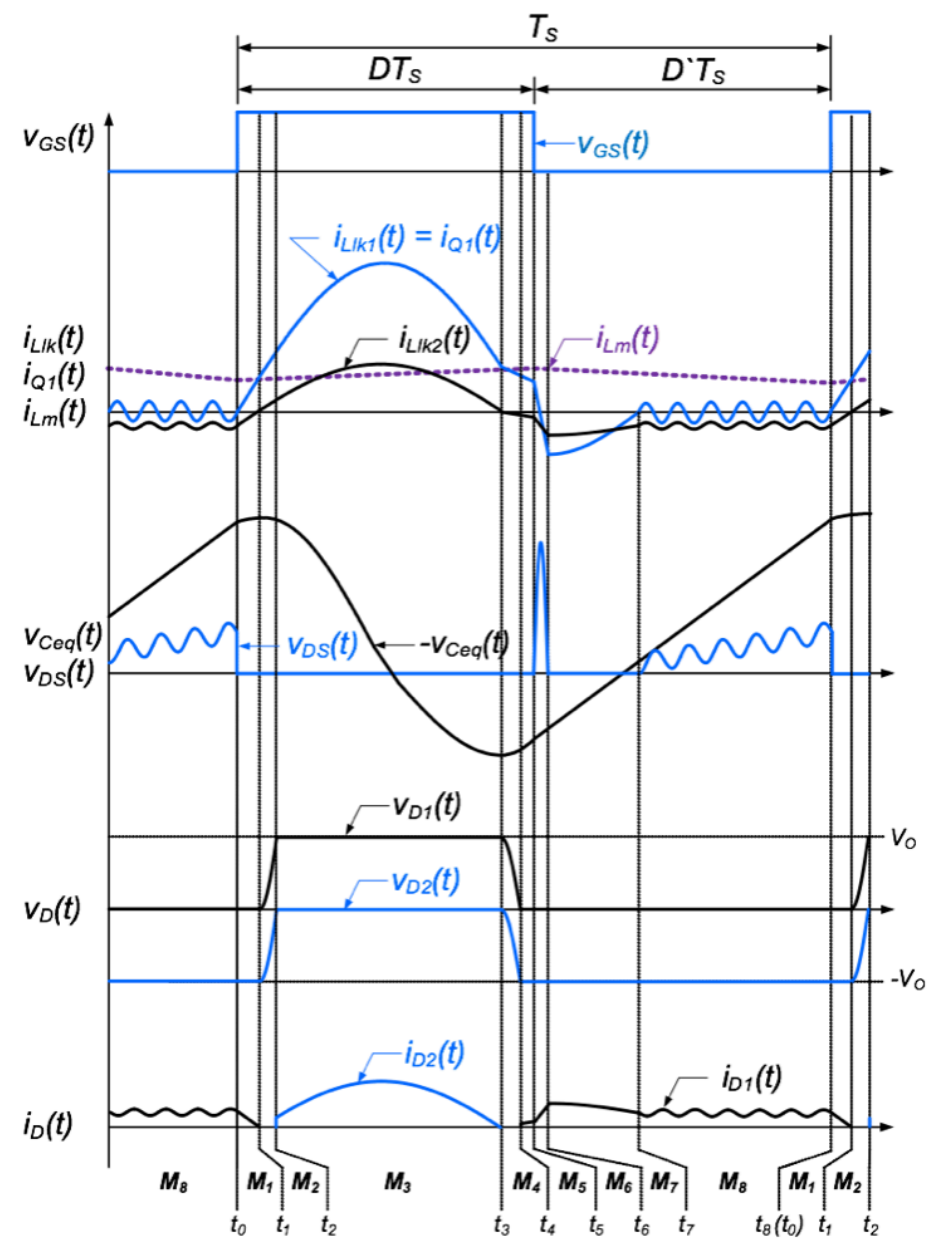

Figure 8. Characteristic waveforms of the proposed SSQR converter for step-up gain.

Mode 1 ( $t_{0}<t \leq t_{1}$ ) [Figure 7a]: This mode begins when $Q_{1}$ turns on. The current flows through the transformer, $Q_{1}, C_{e q}$, and $D_{1}$. The inductances of the transformer resonates with the $C_{e q}$. The $i_{L l k 1}(t)$ and $i_{L l k 2}(t)$ increase according to the quasi-resonant operation. The step-up SSQR converter achieves the quasi-resonant zero-voltage-switching (QR-ZVS). The relation with the currents of the transformer can be expressed as:

$$
i_{L l k 1}(t)=i_{L m}(t)+n \cdot i_{L l k 2}(t)
$$

When $i_{L l k 1}(t)$ meets the magnetizing inductor current $i_{L m}(t)$, the $i_{L l k 2}(t)$ becomes zero. At this time, Mode 1 ends.

Mode $2\left(\boldsymbol{t}_{\mathbf{1}}<\boldsymbol{t} \leq \boldsymbol{t}_{\mathbf{2}}\right)$ [Figure $7 \mathbf{b}$ ]: At $t_{1}, i_{L l k 2}(t)$ changes its direction from negative to positive. $D_{1}$ turns off. $C_{j}$ of $D_{1}$ is charged up and $C_{j}$ of $D_{2}$ is discharged. As a result, the voltage across D1, $V_{D 1}(t)$, increases from zero to $V_{O}$ and the voltage across $\mathrm{D} 2, V_{D 2}(t)$, increases from $-V_{O}$ to zero. $D_{2}$ turns on at $t_{2}$, and Mode 2 ends.

Mode $3\left(t_{2}<t \leq t_{3}\right)$ [Figure $7 \mathrm{c}$ ]: When $D_{2}$ turns on, the resonant current flows through the transformer, $Q_{1}, C_{e q}, D_{2}$, and the load. The difference between $i_{L l k 1}(t)$ and $i_{L m}(t)$ is transferred to the load. When $i_{L l k 1}(t)$ meets $i_{L m}(t)$ again, $i_{L l k 2}(t)$ changes its direction from positive to negative at $t_{3}$. 
Mode $4\left(t_{3}<\boldsymbol{t} \leq \boldsymbol{t}_{4}\right)$ [Figure $7 \mathrm{~d}$ ]: When $i_{L l k 2}(t)$ becomes negative, $D_{2}$ turns off. $C_{j}$ of $D_{2}$ starts to be charged up and $C_{j}$ of $D_{1}$ is to be discharged. As a result, $V_{D 2}(t)$ decreases from zero to $-V_{O}$, and the $V_{D 1}(t)$ decreases from $V_{O}$ to zero. $D_{1}$ turns on at $t_{4}$, and Mode 4 ends.

Mode $5\left(t_{4}<\boldsymbol{t} \leq \boldsymbol{t}_{5}\right)$ [Figure 7e]: When $D_{1}$ turns on, the current flows through the transformer, $Q_{1}, C_{e q}$, and $D_{1}$. After $i_{L l k 1}(t)$ meets $i_{L m}(t)$ again, $i_{L l k 1}(t)$ goes low. At $t_{5}, Q_{1}$ turns off.

Mode $6\left(t_{5}<t \leq t_{6}\right)$ [Figure 7f]: When $Q_{1}$ is turned off, $C_{\text {oss }}$ resonates with the inductances of the transformer. The resonance makes $V_{D S}(t)$ increase and decrease. At this time, $i_{L l k 1}(t)$ changes its direction from positive to negative. When $V_{D S}(t)$ decrease to zero, Mode 6 ends.

Mode $7\left(t_{6}<t \leq t_{7}\right)$ [Figure 7g]: When $V_{D S}(t)$ decrease to zero, the internal anti-parallel diodes of $Q_{1}$ is turned on. The current flows through the transformer, the internal anti-parallel diodes of $Q_{1}, C_{e q}$, and $D_{1} . V_{C_{e q}}(t)$ is applied to the transformer, and then the transformer is reset and $V_{C_{e q}}(t)$ is to be increased. The inductances of the transformer resonates with $C_{e q}$. When $i_{L l k 1}(t)$ becomes zero, Mode 7 ends.

Mode $8\left(t_{7}<\boldsymbol{t} \leq \boldsymbol{t}_{8}\right)$ [Figure 7h]: When $i_{L l k 1}(t)$ becomes zero at $t_{7}$, the current flows through the transformer, $C_{o s s}, C_{e q}$, and $D_{1}$. Since $V_{C_{e q}}(t)$ is applied to the transformer, the transformer is still reset. $V_{C_{e q}}(t)$ then increases. At the same time, the inductances of the transformer resonates with $C_{o s s}$. Consequently, $V_{D S}(t)$ increases with $V_{C_{e q}}(t)$ and resonates with inductances of the transformer.

\section{Experimental Results}

To verify the feasibility of the step-up SSQR converter with the capacitive interface, a prototype SSQR converter has been built with the following specifications: the maximum transferrable power $=50 \mathrm{~W}(100 \mathrm{~V} / 0.5 \mathrm{~A}), V_{I N}=24 \mathrm{~V}$, and $V_{O}=100 \mathrm{~V}$. A picture of the prototype converter is shown in Figure 9, and the components used in the prototype are listed up in Table 1. Note that the tolerance on the capacitances and inductances in the table are less than $10 \%$. To make the capacitance of the small size capacitive interface high, we have used the lead zirconium titanate (PZT) for the dielectric material between the two electrodes, as seen in Figure 9. As aforementioned in Section 2, $C_{e q}$ is half the capacitance of the two capacitors $C_{I 1}$ and $C_{I 2}$. The capacitive interface is composed of four electrodes and two PZT plates (width: $30 \mathrm{~mm}$, length: $70 \mathrm{~mm}$, and thickness: $1 \mathrm{~mm}$ ). Note that these PZT plates represent the two capacitors in Figure 3b. Meanwhile, the amount of power transfer that the current prototype supports may be much larger than the power demand from small IoT devices operating with tiny capacity batteries (e.g., IoT sensor nodes). Due to the implementation difficulty of CPT systems being suitable for such small IoT devices (i.e., chip-level implementation and measurement techniques may be necessary), we focus on demonstrating the feasibility of the proposed converter design implemented with the board-level (relatively large) CPT prototype. As a pioneer design, the proposed CPT converter can be exploited and scaled down to develop high efficient CPT systems that are appropriate for the small IoT devices.

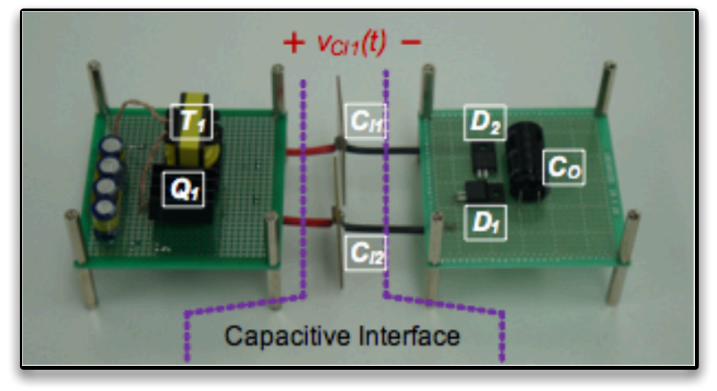

Figure 9. The prototype of the proposed SSQR converter including the capacitive interface made of lead zirconium titanate (PZT). 
Table 1. Component list.

\begin{tabular}{ccc}
\hline Symbol & Description & Value/Part Name \\
\hline$Q_{1}$ & Switch & FDP18N50 \\
$T_{1}$ & Transformer & EER2828 \\
$n$ & Turn ratio & $3.4\left(N_{S} / N_{P}=34 / 10\right)$ \\
$L_{l k 1}$ & Primary inductance & $6.06 \mu \mathrm{H}$ \\
$L_{l k 2}$ & Secondary inductance & $58.47 \mu \mathrm{H}$ \\
$L_{L m}$ & Magnetizing inductance & $82.53 \mu \mathrm{H}$ \\
$P Z T$ & Lead zirconium titanate & $20 \mathrm{nF}$ \\
$C_{I 1}$ & Capacitance of the capacitive interface 1 & $20 \mathrm{nF}$ \\
$C_{I 2}$ & Capacitance of the capacitive interface 2 & $10 \mathrm{nF}$ \\
$C_{e q}$ & Equivalent capacitance of the capacitive interface & PI PIC151 $\left(\epsilon_{r}=2400,30 \mathrm{~mm}\right)$ \\
$D_{1}$ and $D_{2}$ & Diodes & MBRF20H150CTG \\
\hline
\end{tabular}

Figure 10 shows the resulting waveforms of the gate signal $V_{G S 1}(t)$, the primary leakage inductor current $i_{L l k 1}(t)$, the voltage across a switch $V_{D S}(t)$, and the voltage across interface $1 V_{C I 2}(t)$. The laboratory prototype SSQR converter utilizes the resonance between the transformer $T_{1}$ and the capacitive interface $C_{I 1}$ and $C_{I 2}$ during the switch conducting interval $D T_{S}$. The resonant operation is started and finished in $D T_{S}$. When the switch is turned on, the primary leakage inductor current $i_{L l k 1}(t)$ is almost zero. For this reason, the turned-on loss of the switch is very low. Since the resonant operation shapes the current sinusoidal, and the primary leakage inductor current $i_{L l k 1}(t)$ is low when the switch is turned off, the turn-off loss of the switch is reduced. To regulate the output voltage $V_{O}$, a PWM control has been used for different load conditions.

To show the characteristics of the capacitive interface, the film capacitor $C_{r}$, instead of the capacitive interface, has been also used during the experimental work, as the resonant capacitor in the proposed step-up SSQR converter. Then, we have measured the resulting efficiency of the converter for each case and have performed comparison. When the film capacitor is used, the operation of the prototype converter results in the similar switching frequency $f_{S}$ and duty ratio $D$ to those from the prototype converter with the capacitive interface. Therefore, the waveforms in Figure 11 look similar to the waveforms in Figure 10. As shown in Figure 12, the efficiency of the step-up SSQR converter with the film capacitor for $20 \%, 50 \%$, and $100 \%$ load conditions are $95.52 \%, 95.28 \%$, and $91.44 \%$, respectively. On the other hand, when the capacitive interface is applied instead of the film capacitor, the efficiency of the step-up SSQR converter is reduced as seen in Figure 12. This efficiency degradation may be resulted from the high ESRs of the capacitive interface. Although the step-up turn ratio $n$ of the transformer enables the capacitive interface to drive relatively low current, increasing load current may induce the higher conduction loss due to the high ESRs of the capacitive interface. The rising conduction loss according to increasing load current can result in more heat generation. In other words, the temperature of the capacitive interface may increase as the the load current increases, which is shown in Figure 13. However, the advantages of the proposed SSQR converter far outweigh the disadvantages, in that it still achieves the high efficiency at normal operating conditions with less number of components than the traditional CPT converters. For example, Table 2 shows the comparison results between our proposed method and other methods to achieve the maximum efficiency of a CPT system. 


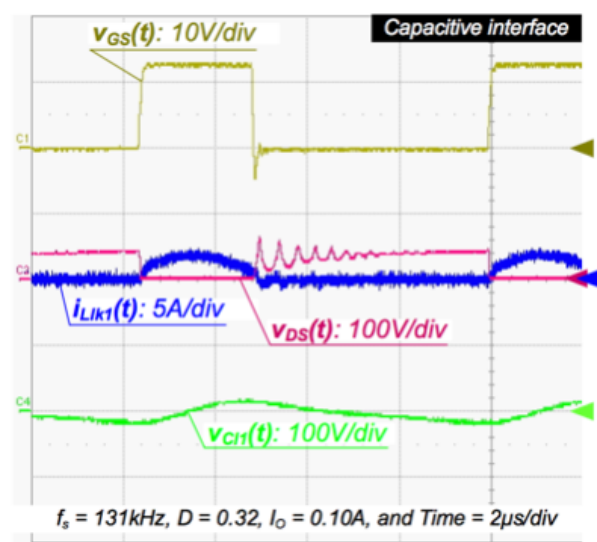

(a)

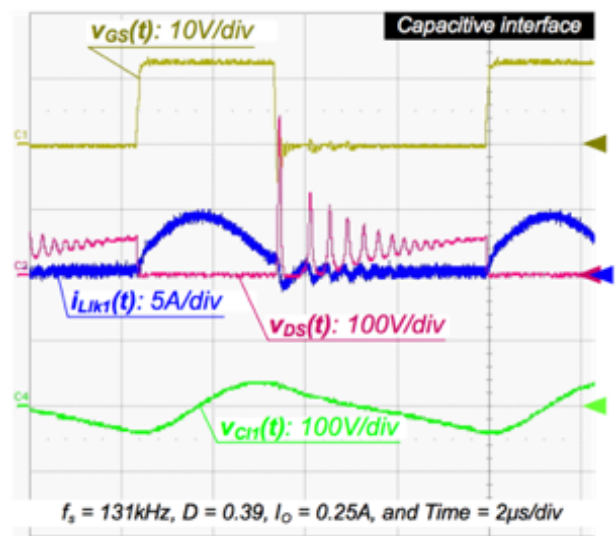

(b)

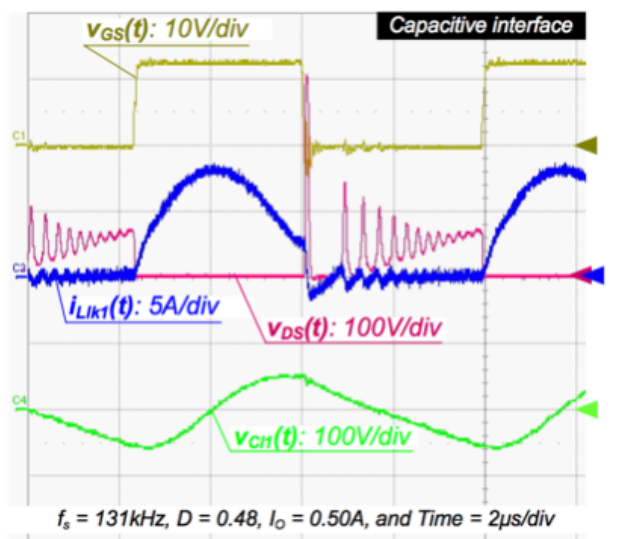

(c)

Figure 10. Experimental waveforms of gate signal $V_{G S 1}(t)$, primary leakage inductor current $i_{L l k 1}(t)$, voltage across a switch $V_{D S}(t)$, and voltage across interface $1 V_{C I 1}(t)$, when (a) $20 \%$; (b) 50\%; and (c) $100 \%$ load is applied.

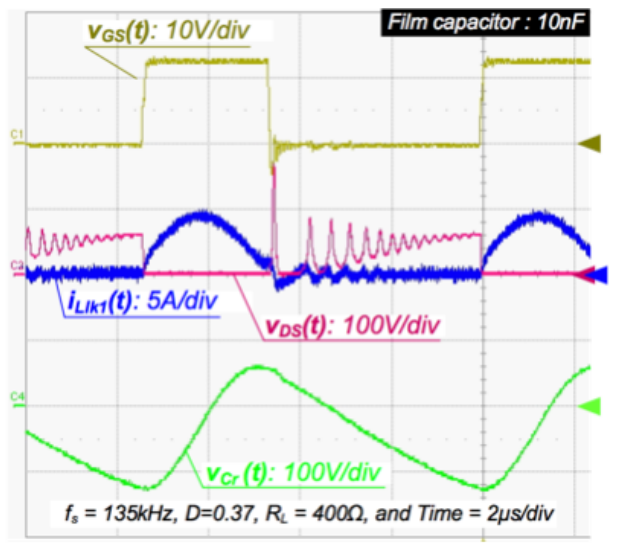

Figure 11. Experimental waveforms of $V_{G S 1}(t), i_{L l k 1}(t), V_{D S}(t)$, and $V_{C I 1}(t)$, when $50 \%$ load is applied. A film capacitor is used in this case. 


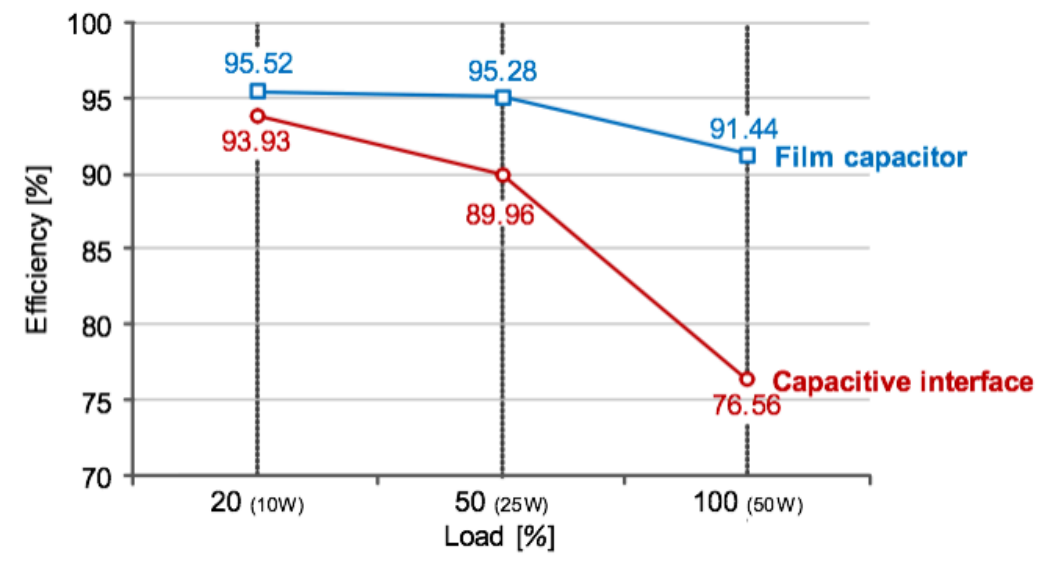

Figure 12. Measured efficiency according to various load conditions: the efficiency is measured from the prototype converter that equips the film capacitor or the capacitive interface.

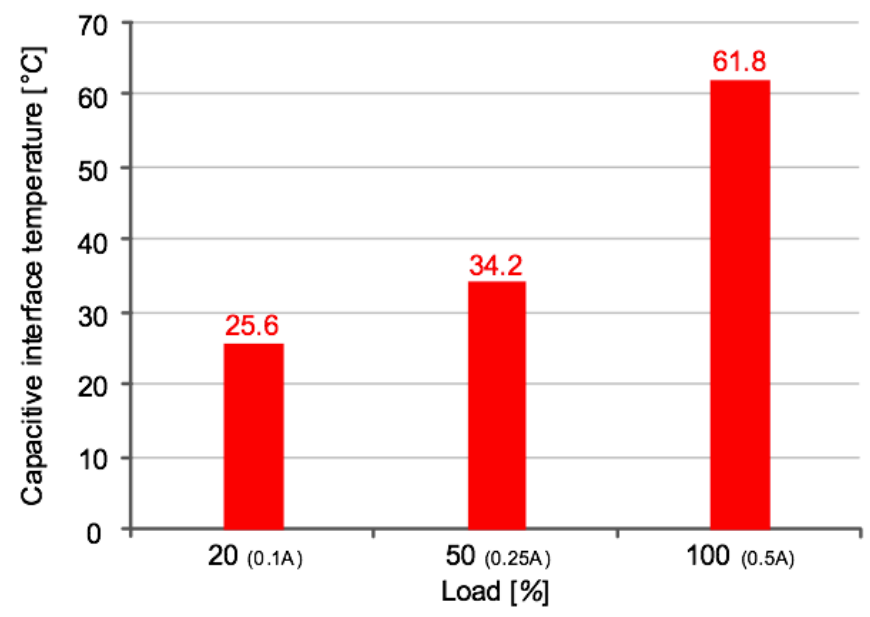

Figure 13. Measured temperature of the capacitive interface under various load conditions.

Table 2. Comparison between our proposed method and other methods.

\begin{tabular}{cccc}
\hline Max. Transferred Power (W) & Size $\left(\mathbf{m m}^{\mathbf{2}}\right)$ & Max. Efficiency (\%) & Source of the Method \\
\hline 3.8 & $60 \times 100$ & 83 & {$[23]$} \\
23 & unreported & 80 & {$[8]$} \\
260 & $100 \times 200$ & 79 & {$[27]$} \\
50 & $30 \times 70$ & 93 & the proposed \\
\hline
\end{tabular}

\section{Conclusions}

Conventional CPT converters that transfer power through the capacitive interface have drawbacks that their efficiencies are quite low, and their implementation costs are expensive due to the high component count. More precisely, the most CPT converters suffer from the small capacitance of the capacitive interface, which makes the CPT converters operate at high resonant frequency (thereby switching loss is increased) and have high ESR (thereby conduction loss is increased), finally operating with low efficiency. In addition, a single CPT converter requires a full-bridge inverter, a resonant inductor, capacitive interface, a full-bridge rectifier, and an output filter, thus the implementation cost is nothing to sneeze at. 
In this paper, a high-efficiency and cost-effective step-up SSQR converter is proposed for CPT. The capacitive interface is used as the resonant capacitor of the SSQR converter for low component counts in CPT applications. The proposed SSQR converter operates with low operating frequency (thereby switching loss is decreased) and achieves the minimum driving current that flows into the capacitive interface (thereby conduction loss is decreased). Plus, the proposed SSQR converter gets rid of an RCD snubber that is unnecessary for step-up operation, which results in power saving as well as implementation cost reduction. The validity of the step-up SSQR converter has been verified in the prototype system. For fair comparison, the capacitive interface as well as the film capacitor is used as the resonant capacitor in the proposed converter. According to the intensive and comparative experimental work based on the prototype, the proposed step-up SSQR converter demonstrates its superiority for CPT systems, in that it achieves high efficiency with the less component count thanks to the low resonant frequency, the low capacitive interface current, and the elimination of RCD snubber. The experimental results show that the maximum efficiency of $93.93 \%$ is achieved at $20 \%$ load condition.

Acknowledgments: This research is supported by Basic Science Research Program through the National Research Foundation of Korea (NRF) partially funded by the Ministry of Education (2017R1D1A1B03027911) and the Ministry of Science, ICT \& Future Planning (2015R1C1A1A01051992).

Author Contributions: 1l-Oun Lee and Woojoo Lee were the main researchers who initiated and organized research reported in the paper, and all authors including Joongheon Kim were responsible for analyzing the simulation results and writing the paper.

Conflicts of Interest: The authors declare no conflict of interest.

\section{References}

1. Choi, B.H.; Thai, V.X.; Lee, E.S.; Kim, J.H.; Rim, C.T. Dipole-Coil-Based Wide-Range Inductive Power Transfer Systems for Wireless Sensors. IEEE Trans. Ind. Electron. 2016, 63, 3158-3167.

2. Jawad, A.M.; Nordin, R.; Gharghan, S.K.; Jawad, H.M.; Ismail, M. Opportunities and Challenges for Near-Field Wireless Power Transfer: A Review. Energies 2017, 10, 1022.

3. Kurs, A.; Karalis, A.; Moffatt, R.; Joannopoulos, J.D.; Fisher, P.; Soljačić, M. Wireless Power Transfer via Strongly Coupled Magnetic Resonances. Science 2007, 317, 83-86.

4. Sample, A.P.; Meyer, D.T.; Smith, J.R. Analysis, Experimental Results, and Range Adaptation of Magnetically Coupled Resonators for Wireless Power Transfer. IEEE Trans. Ind. Electron. 2011, 58, 544-554.

5. Sodagar, A.M.; Amiri, P. Capacitive coupling for power and data telemetry to implantable biomedical microsystems. In Proceedings of the 2009 4th International IEEE/EMBS Conference on Neural Engineering, Antalya, Turkey, 29 April-2 May 2009; pp. 411-414.

6. Liu, C.; Hu, A.P.; Budhia, M. A generalized coupling model for Capacitive Power Transfer systems. In Proceedings of the Annual Conference on IEEE Industrial Electronics Society, Glendale, AZ, USA, 7-10 November 2010; pp. 274-279.

7. Kline, M. Capacitive Power Transfer. Master's Thesis, EECS Department, University of California, Berkeley, CA, USA, 2010.

8. Theodoridis, M.P. Effective Capacitive Power Transfer. IEEE Trans. Power Electron. 2012, 27, 4906-4913.

9. Karagozler, M.E.; Goldstein, S.C.; Ricketts, D.S. Analysis and Modeling of Capacitive Power Transfer in Microsystems. IEEE Trans. Circuits Syst. I Regul. Pap. 2012, 59, 1557-1566.

10. Dai, J.; Ludois, D.C. Single Active Switch Power Electronics for Kilowatt Scale Capacitive Power Transfer. IEEE J. Emerg. Sel. Top. Power Electron. 2015, 3, 315-323.

11. Ramos, I.; Afridi, K.; Estrada, J.A.; Popovi, Z. Near-field capacitive wireless power transfer array with external field cancellation. In Proceedings of the IEEE Wireless Power Transfer Conference (WPTC), Aveiro, Portugal, 5-6 May 2016; pp. 1-4.

12. Minnaert, B.; Stevens, N. Conjugate Image Theory Applied on Capacitive Wireless Power Transfer. Energies $2017,10,46$. 
13. Hu, A.P.; Liu, C.; Li, H.L. A Novel Contactless Battery Charging System for Soccer Playing Robot. In Proceedings of the 2008 15th International Conference on Mechatronics and Machine Vision in Practice, Auckland, New Zealand, 2-4 December 2008; pp. 646-650.

14. Zheng, H.; Tnay, K.; Alami, N.; Hu, A.P. Contactless power couplers for respiratory devices. In Proceedings of the 2010 IEEE/ASME International Conference on Mechatronic and Embedded Systems and Applications, Qingdao, China, 15-17 July 2010; pp. 155-160.

15. Ludois, D.C.; Reed, J.K.; Hanson, K. Capacitive Power Transfer for Rotor Field Current in Synchronous Machines. IEEE Trans. Power Electron. 2012, 27, 4638-4645.

16. Ludois, D.C.; Erickson, M.J.; Reed, J.K. Aerodynamic Fluid Bearings for Translational and Rotating Capacitors in Noncontact Capacitive Power Transfer Systems. IEEE Trans. Ind. Appl. 2014, 50, 1025-1033.

17. Han, K.; Lee, J.J.; Lee, J.; Lee, W.; Pedram, M. TEI-NoC: Optimizing Ultra-Low Power NoCs Exploiting the Temperature Effect Inversion. IEEE Trans. Comput. Aided Des. Integr. Circuits Syst. 2017, doi:10.1109/ TCAD.2017.2693269.

18. Lee, W.; Wang, Y.; Pedram, M. VRCon: Dynamic Reconfiguration of Voltage Regulators in a Multicore Platform. In Proceedings of the Conference on Design, Automation \& Test in Europe (DATE '14), Dresden, Germany, 24-28 March 2014.

19. Lee, W.; Wang, Y.; Pedram, M. Optimizing a Reconfigurable Power Distribution Network in a Multicore Platform. IEEE Trans. Comput. Aided Des. Integr. Circuits Syst. 2015, 34, 1110-1123.

20. Lee, W.; Wang, Y.; Shin, D.; Chang, N.; Pedram, M. Power Conversion Efficiency Characterization and Optimization for Smartphones. In Proceedings of the 2012 ACM/IEEE International Symposium on Low Power Electronics and Design (ISLPED '12), Redondo Beach, CA, USA, 30 July-1 August 2012; pp. $103-108$.

21. Lee, W.; Wang, Y.; Shin, D.; Chang, N.; Pedram, M. Optimizing the Power Delivery Network in a Smartphone Platform. IEEE Trans. Comput. Aided Des. Integr. Circuits Syst. 2014, 33, 36-49.

22. Lee, W.; Wang, Y.; Shin, D.; Nazarian, S.; Pedram, M. Design and optimization of a reconfigurable power delivery network for large-area, DVS-enabled OLED displays. In Proceedings of the 2015 IEEE/ACM International Symposium on Low Power Electronics and Design (ISLPED), Rome, Italy, 22-24 July 2015; pp. 159-164.

23. Kline, M.; Izyumin, I.; Boser, B.; Sanders, S. Capacitive power transfer for contactless charging. In Proceedings of the IEEE Applied Power Electronics Conference and Exposition (APEC), Fort Worth, TX, USA, 6-11 March 2011; pp. 1398-1404.

24. Liu, C.; Hu, A.; Nair, N.K. Modeling and analysis of a capacitively coupled contactless power transfer system. IET Power Electron. 2011, 4, 808-815.

25. Fiore, R. Complete Circuit Designer's Notebook. American Technical Ceramics Corp. Available online: http:/ / www.atceramics.com/ (accessed on 1 September 2017).

26. Park, K.B.; Kim, C.E.; Moon, G.W.; Youn, M.J. PWM Resonant Single-Switch Isolated Converter. IEEE Trans. Power Electron. 2009, 24, 1876-1886.

27. Su, Y.G.; Xie, S.Y.; Hu, A.P.; Tang, C.S.; Zhou, W.; Huang, L. Capacitive Power Transfer System with a Mixed-Resonant Topology for Constant-Current Multiple-Pickup Applications. IEEE Trans. Power Electron. 2017, 32, 8778-8786.

(C) 2017 by the authors. Licensee MDPI, Basel, Switzerland. This article is an open access article distributed under the terms and conditions of the Creative Commons Attribution (CC BY) license (http:// creativecommons.org/licenses/by/4.0/). 